\title{
Effect of hydroxyl group position in flavonoids on inducing single-stranded DNA damage mediated by cupric ions
}

\author{
SUAD ELMEGERHI ${ }^{1}$, CATHY SU ${ }^{2}$, DYLAN JOHN BUGLEWICZ ${ }^{2}$, \\ YASUSHI AIZAWA ${ }^{3}$ and TAKAMITSU A KATO ${ }^{1,2}$ \\ ${ }^{1}$ Cell and Molecular Biology Program, and ${ }^{2}$ Department of Environmental and Radiological Health Sciences, \\ Colorado State University, Fort Collins, CO 80523, USA; ${ }^{3}$ Research and Development Group, \\ Toyo Sugar Refining Co., Ltd., Tokyo 103-0046, Japan
}

Received January 6, 2018; Accepted March 22, 2018

DOI: $10.3892 /$ ijmm.2018.3615

\begin{abstract}
Quercetin has been demonstrated to produce DNA damage in the presence of metal ions. In the present study, 7 natural and 5 semi-synthetic glycosylated flavonoids were utilized to investigate the cupric ion $\left(\mathrm{Cu}^{2+}\right)$-dependent DNA damage in vitro. The reaction mixture, containing single-stranded DNA, different concentrations of flavonoids and cupric ion in the buffer, was incubated at three different temperatures. DNA damage was then assessed by gel electrophoresis followed by densitometric analysis. The reaction mixture with quercetin at 4,20 and $54^{\circ} \mathrm{C}$ induced DNA damage in a concentration- and temperature-dependent manner. Furthermore, only the reaction at $54^{\circ} \mathrm{C}$ resulted in DNA damage in flavonoids with glucosyl substitution of the hydroxyl group at the 3-position on the $\mathrm{C}$ ring in quercetin. By contrast, loss of the hydroxyl group at the 3-position on the $\mathrm{C}$ ring, or at the 3'- or 4'-position on the B ring of quercetin, did not portray DNA damage formation at the investigated experimental temperatures. In addition, the experimental results suggested that the hydroxyl group at the 3-position on the $\mathrm{C}$ ring produced the strongest capability to induce DNA damage in the presence of cupric ions. Furthermore, hydroxyl groups at the 3'- or 4'-position on the B ring were only able to induce DNA damage at higher temperatures, and were less efficient in comparison with the hydroxyl group at the 3-position on the $\mathrm{C}$ ring. Cupric ion chelating capacity was also assessed with spectroscopic analysis, and quercetin presented the largest chelating capacity among the tested flavonoids. Hydroxyl radical formation was assessed with a luminol reaction, and quercetin presented faster consumption of luminol. These results suggest that the 3-position hydroxyl group of the $\mathrm{C}$ ring is required to induce DNA damage at low temperatures.
\end{abstract}

Correspondence to: Professor Takamitsu A Kato, Department of Environmental and Radiological Health Sciences, Colorado State University, 1618 Campus Delivery, Fort Collins, CO 80523, USA

E-mail: takamitsu.kato@colostate.edu

Key words: quercetin, cupric ion, DNA scission
Furthermore, the results of the present study also indicated that the presence of cupric ions will decrease the activity of the glycosylated quercetins, in terms of their ability to induce DNA damage.

\section{Introduction}

Flavonoids are naturally occurring polyphenolic metabolites in plants that serve significant roles in traditional medicine and as food additives (1-3). Glycosylated forms of numerous flavonoids are produced in order to alter their chemical properties, such as their bioavailability and water solubility (4). Flavonoids without glycosylation are known as aglycones, which are often more reactive forms of flavonoids and present greater antioxidant capacity, as well as cellular toxicity, compared with the glycosylated flavonoids $(5,6)$. Both aglycone and glycosylated forms of flavonoids are recognized to be beneficial in regard to anti-tumorigenicity and anti-mutagenicity, due to their antioxidant and radical scavenging effects.

Numerous flavonoids, including quercetin, have been previously reported to induce mutation frequency in bacterial systems and chromosome aberrations in mammalian cells (7-10). This mutagenic potential may be associated with the ability of flavonoids to produce hydroxyl radicals, resulting in DNA breaks $(11,12)$. In the presence of specific metal ions, flavonoids can cause DNA scission, but rarely DNA double-strand breaks, resulting from the interaction of hydrogen peroxide with metal ions (12). This hydroxyl radical production is coupled with the reduction of cupric ions to cuprous ions (from $\mathrm{Cu}^{2+}$ to $\mathrm{Cu}^{1+}$ ). Quercetin forms chelating complexes with cupric ions and portrays broad biological activities (13-16). This cupric ion and the quercetin complex interact at the $3^{\prime}$ and 4 ' hydroxyl groups on the $\mathrm{B}$ ring, and the 3-hydroxyl group and 4-oxygen residue on the A ring (17). Furthermore, the ability to produce hydroxyl radicals and to induce DNA breaking activity has also been reported for other compounds that are structurally associated with quercetin, including fisetin, baicalein, taxifolin and curcumin with cupric ions $(18,19)$, as well as epigallocatechin gallate and ferrous ions (20). However, the exact mechanisms underlying the formation of hydroxyl radicals from quercetin and its effects on glycosylation of flavonoids remain unclear. 
The present study utilizes a total of 7 natural and 5 recently semi-synthesized novel flavonoids to identify the specific molecular mechanisms required to induce DNA scissions in the presence of cupric ions. To analyze DNA scission formation in an in vitro gel electrophoresis system, three aglycones and their glycosylated flavonoids were reacted in the presence of cupric ions at different temperatures.

\section{Materials and methods}

Chemicals. All natural and synthetic flavonoids were obtained from Tokyo Sugar Refining Co., Ltd. (Tokyo, Japan). The tested compounds, including three aglycone flavonoids (quercetin, naringenin and hesperetin) and their glycosylated flavonoids are summarized in Fig. 1. Quercetin is an active aglycone form of isoquercetin, rutin, maltooligosyl-isoquercetin, monoglucosyl-rutin and maltooligosyl-rutin. The glycosylated form of quercetin loses the 3-position hydroxyl group on the B ring by glycosylation. Compared with quercetin, naringenin does not contain a hydroxyl group at the 3-position on the $\mathrm{B}$ ring nor at the 3 '-position on the $\mathrm{C}$ ring. In addition, compared with quercetin, hesperetin does not contain a hydroxyl group at the 3-position on the $\mathrm{C}$ ring nor at the 4'-position on the $\mathrm{C}$ ring. Glycosylated naringenin and hesperetin lost the hydroxyl group at the 7-position on the A ring by glycosylation and are chemically inactive. All flavonoids were prepared by dissolving in dimethyl sulfoxide (Thermo Fisher Scientific, Inc., Waltham, MA, USA) at the concentration of $1 \mathrm{mM}$ as a stock solution. M13mp18 single-stranded DNA $(250 \mu \mathrm{g} / \mathrm{ml})$ was purchased from New England BioLabs, Inc. (Ipswich, MA, USA). Double-stranded lambda phage DNA $(0.46 \mu \mathrm{g} / \mu \mathrm{l})$ was purchased from Nippon Gene Co., Ltd. (Tokyo, Japan). Copper chloride dehydrate was purchased from Sigma-Aldrich (Merck KGaA, Darmstadt, Germany).

DNA scission reaction. A total of $20 \mu 1$ reaction solution was used, containing $1.5 \mathrm{ng} / \mu \mathrm{l}$ single-stranded DNA or $1.25 \mathrm{ng} / \mu \mathrm{l}$ of double-stranded DNA, $10 \mathrm{mM}$ Tris- $\mathrm{HCl}, 0.2 \mathrm{mM} \mathrm{CuCl}_{2}$ and various concentrations of flavonoids $(0.1,1,10$ and $100 \mu \mathrm{M})$. The reaction mixtures were incubated at different temperatures $\left(4^{\circ} \mathrm{C}, 20^{\circ} \mathrm{C}, 37^{\circ} \mathrm{C}\right.$ or $54^{\circ} \mathrm{C}$ ) for $1 \mathrm{~h}$. Next, $4 \mu \mathrm{l}$ of $6 \mathrm{X}$ loading dye, containing $15 \%$ Ficoll, $10 \%$ glycerol, $0.25 \%$ bromophenol blue, and $0.25 \%$ xylene cyanol in water, was added to the mixture and electrophoresis was conducted on $1 \%$ agarose gel stained with ethidium bromide with $1 \mathrm{X}$ Tris-acetate-EDTA buffer at $100 \mathrm{~V}$ for $1 \mathrm{~h}$. Subsequent to electrophoresis, destaining of ethidium bromide was performed, and a gel image was obtained by ChemiDoc XRS system (Bio-Rad Laboratories, Inc., Hercules, CA, USA) via Image Lab software (Bio-Rad Laboratories, Inc.). Intact M13mp18 single-stranded DNA presented two bands, indicative of the circular and linear form of DNA. The DNA intensity of the circular form was obtained by Image Lab, while the fraction of intact DNA was calculated as follows:

$$
\text { Fraction of intact DNA }=\frac{\text { Amount of circular DNA with flavonoids }}{\text { Amount of circular DNA without flavonoids }}
$$

Half maximal inhibitory concentration $\left(\mathrm{IC}_{50}\right)$ values, which refer to the specific concentrations required to induce $50 \%$ of DNA break, were obtained from sigmoidal regression curves obtained by Prism 6 software (GraphPad Software, Inc., La Jolla, CA, USA).

Absorption spectrum analysis. In order to investigate the cupric ion chelating capability of flavonoids, the flavonoids and cupric ions were mixed at rations of 1:2, 1:1 and 2:1, and absorbance values from 230 to $430 \mathrm{~nm}$ were obtained via a Nanodrop spectrophotometer (Thermo Fisher Scientific, Inc.). According to the peak shifting and the reduction of peak height, the chelating capacity of cupric ions was estimated $(14,15,17)$.

Oxidation of luminol. In order to investigate the potential mechanisms underlying the induction of DNA damages, oxidative capacity analysis was conducted. In total, $100 \mu \mathrm{l}$ of reaction solution, including $20 \mu \mathrm{l}$ enhanced chemiluminescence solution (Thermo Fisher Scientific, Inc.), $2 \mu \mathrm{l}$ of $100 \mu \mathrm{M}$ flavonoids and $2 \mu \mathrm{l}$ of $100 \mu \mathrm{M} \mathrm{CuCl}_{2}$, were mixed. Subsequently, the arbitrary relative luminescence unit (RLU) was measured immediately until 3 min after mixing via a Lumat LB9507 luminometer (Berthold Technologies, Oak Ridge, TN, USA). RLU values were plotted with the exponential decay model, and half time values were obtained.

Statistical analysis. Statistical calculations were preformed via Prism 6 software. Two-way analysis of variance (ANOVA) and Student's t-test were conducted. P-values of $<0.05$ were considered to indicate differences that were statistically significant.

\section{Results}

DNA damage formation. Fig. 2 demonstrates the DNA damage subsequent to reaction with quercetin in the absence or presence of cupric ions at $37^{\circ} \mathrm{C}$. Without cupric ions in the reaction mixture, DNA damage was not detected from the single-stranded and double-stranded DNA. Upon the incorporation of cupric ions in the reaction mixture, single-stranded DNA was degraded, whereas double-stranded DNA was not affected. Therefore, quercetin without cupric ions did not produce DNA damage. In addition, DNA damage produced by quercetin and cupric ions mainly involved single-strand break rather than double-strand break.

Fig. 3 shows the fraction of intact DNA following reaction with glycosylated flavonoids in the presence of cupric ions in the reaction mixture. In the presence of $0.2 \mathrm{mM} \mathrm{CuCl}_{2}$ in the reaction mixture, quercetin and its glycosylated flavonoids induced DNA breaks in a concentration and temperature dependent manner. Less intact DNA was observed following reaction with high concentration of flavonoids, and high temperature efficiently induced DNA damages.

Quercetin was the only flavonoid capable of inducing a DNA break in reactions performed at 4 and $20^{\circ} \mathrm{C}$. Furthermore, the reaction at $20^{\circ} \mathrm{C}$ induced a greater degree of DNA damage as compared with the reaction at $4^{\circ} \mathrm{C}$. The $\mathrm{IC}_{50}$ values of quercetin at 4 and $20^{\circ} \mathrm{C}$ were 47.7 and $20.5 \mu \mathrm{M}$, respectively, while the reaction at $54^{\circ} \mathrm{C}$ reduced the $\mathrm{IC}_{50}$ value to $2.6 \mu \mathrm{M}$. By contrast, all quercetin glycosides, including isoquercetin, rutin, monoglucosyl rutin, maltooligosyl-rutin and maltooligosyl-isoquercetin, failed to induce any DNA damage following 4 and $20^{\circ} \mathrm{C}$ reactions under the tested conditions. However, these glucosyl flavonoids did induce DNA 
A<smiles>COc1cc(O)ccc1-c1ccc(O)c(O)c1</smiles>

D

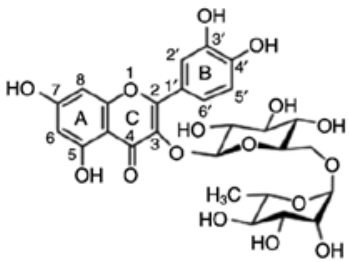

G<smiles>COc1ccc(C2CC(=O)c3c(O)cc(O)cc3O2)cc1O</smiles>

$J$

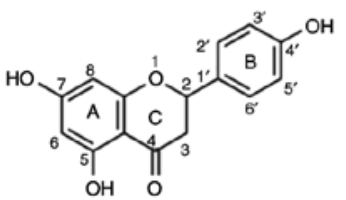

B

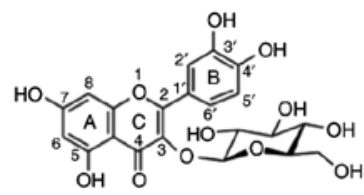

E

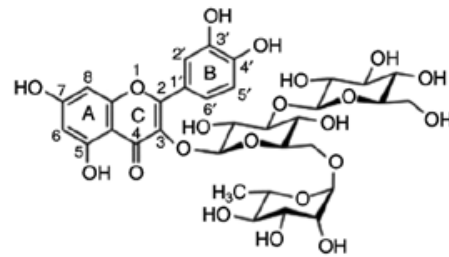

$\mathrm{H}$

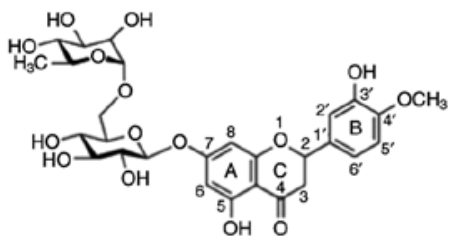

$\mathrm{K}$

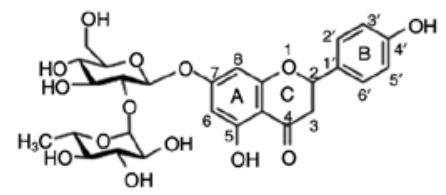

C

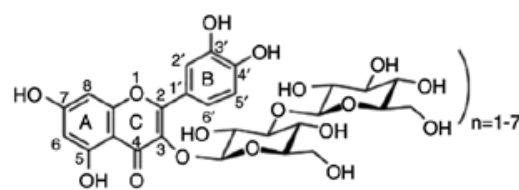

$\mathrm{F}$

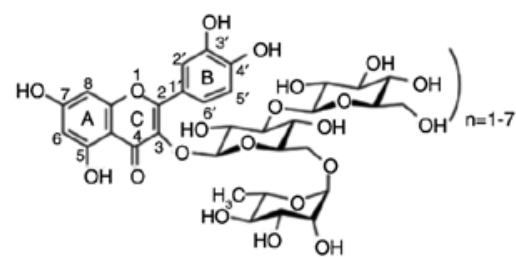

I
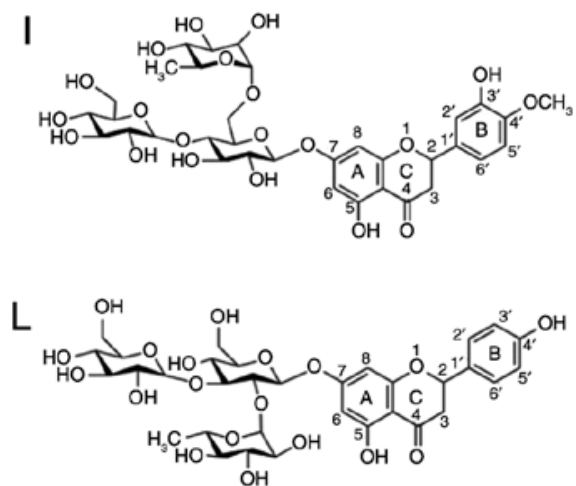

Figure 1. Structure of flavonoids. (A) Quercetin, (B) isoquercetin, (C) rutin, (D) monoglucosyl-rutin, (E) maltooligosyl-isoquercetin, (F) maltooligosyl rutin, $(\mathrm{G})$ hesperetin, $(\mathrm{H})$ hesperidin, $(\mathrm{I})$ monoglucosyl hesperidin, $(\mathrm{J})$ naringenin, $(\mathrm{K})$ naringin, and $(\mathrm{L})$ monoglucosyl naringin.

damage when reactions were conducted at $54^{\circ} \mathrm{C}$ for $1 \mathrm{~h}$. The $\mathrm{IC}_{50}$ values were $7.7 \mu \mathrm{M}$ for isoquercetin, $3.4 \mu \mathrm{M}$ for rutin, $9.5 \mu \mathrm{M}$ for monoglucosyl rutin, $4.5 \mu \mathrm{M}$ for maltooligosyl rutin and $12.1 \mu \mathrm{M}$ for maltooligosyl isoquercetin. This indicates that glycosyl modifications at the 3-position on the $\mathrm{C}$ ring of quercetin suppressed the DNA scission ability of quercetin at 4 and $20^{\circ} \mathrm{C}$. However, DNA scission capacity was not altered with glycosylation at the 3-position on the $\mathrm{C}$ ring of quercetin at $54^{\circ} \mathrm{C}$. Therefore, increased glucosyl modifications did not contribute toward DNA degradation. In other words, the hydroxyl groups of glucosyl residues did not portray any contributions towards DNA damage.

In order to clarify which position of the hydroxyl group is responsible for inducing DNA damage, two other flavone aglycones, hesperetin and naringenin, and their glycosylated flavonoids were also tested in the same system to assess hydroxyl radical formation by DNA damage observation (Fig. 4). Hesperetin, naringenin and their glycosylated flavonoids, which do not have hydroxyl groups at the 3-position on the $\mathrm{C}$ ring nor at the 3'- or 4'-position on the $\mathrm{B}$ ring, were incapable of producing any detectable single-stranded DNA damage in the reaction at any tested temperature. Therefore, this indicates that the presence of hydroxyl groups at the 3'- and 4'-positions is required for the induction of DNA damage at $54^{\circ} \mathrm{C}$. Furthermore, flavone glycosides did not cause any DNA damage, confirming that the hydroxyl groups of glucosyl residue have no effect on DNA damage.

Chelating capacity. Absorption spectrum alterations, shown in Fig. 5A-L, occurred when flavonoids and cupric ions were

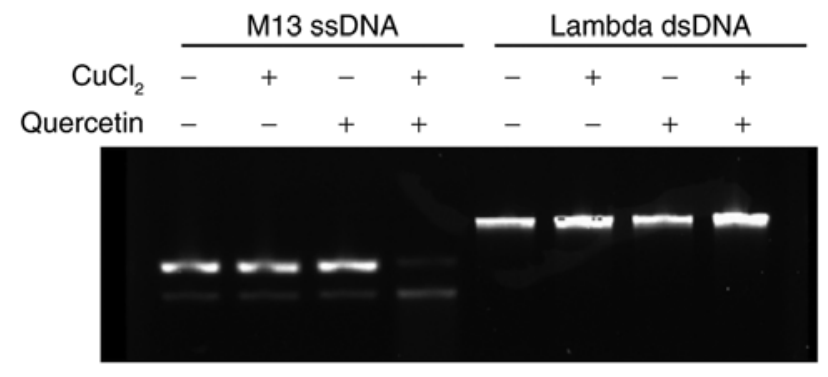

Figure 2. Gel image indicating DNA damage after 1-h reaction at $37^{\circ} \mathrm{C}$. M13 ssDNA (upper bands indicate circular intact DNA and lower bands indicate linear DNA) and lambda dsDNA were treated with or without $0.2 \mathrm{mM} \mathrm{CuCl}_{2}$ and $100 \mu \mathrm{M}$ quercetin in $10 \mathrm{mM}$ Tris- $\mathrm{HCl}$ solution. ss, single-stranded; ds, double-stranded.

mixed in different ratios (1:2, 1:1 and 2:1). Quercetin and its glycosides displayed peaks at $\sim 250$ and $370 \mathrm{~nm}$, as previously described $(14,15,17)$. Upon addition of cupric ions, the first peak at $250 \mathrm{~nm}$ skewed to the left and the size of the second peak was reduced. The reduction in the second peak was the greatest for quercetin. Naringenin, hesperetin and their glycosides presented the first peak at $250 \mathrm{~nm}$ and the second peak at $290 \mathrm{~nm}$. As observed in quercetin and its glycosides, the first peak was shifted toward the left and the second peak size was decreased in the presence of cupric ions. The reduction ratio of the second peak was used for assessment of cupric ion chelating capacity, and the results are summarized in Fig. 5M. Quercetin demonstrated $>6$-fold reduction in the absorbance peak height in the presence of cupric ions. The peak heights of 

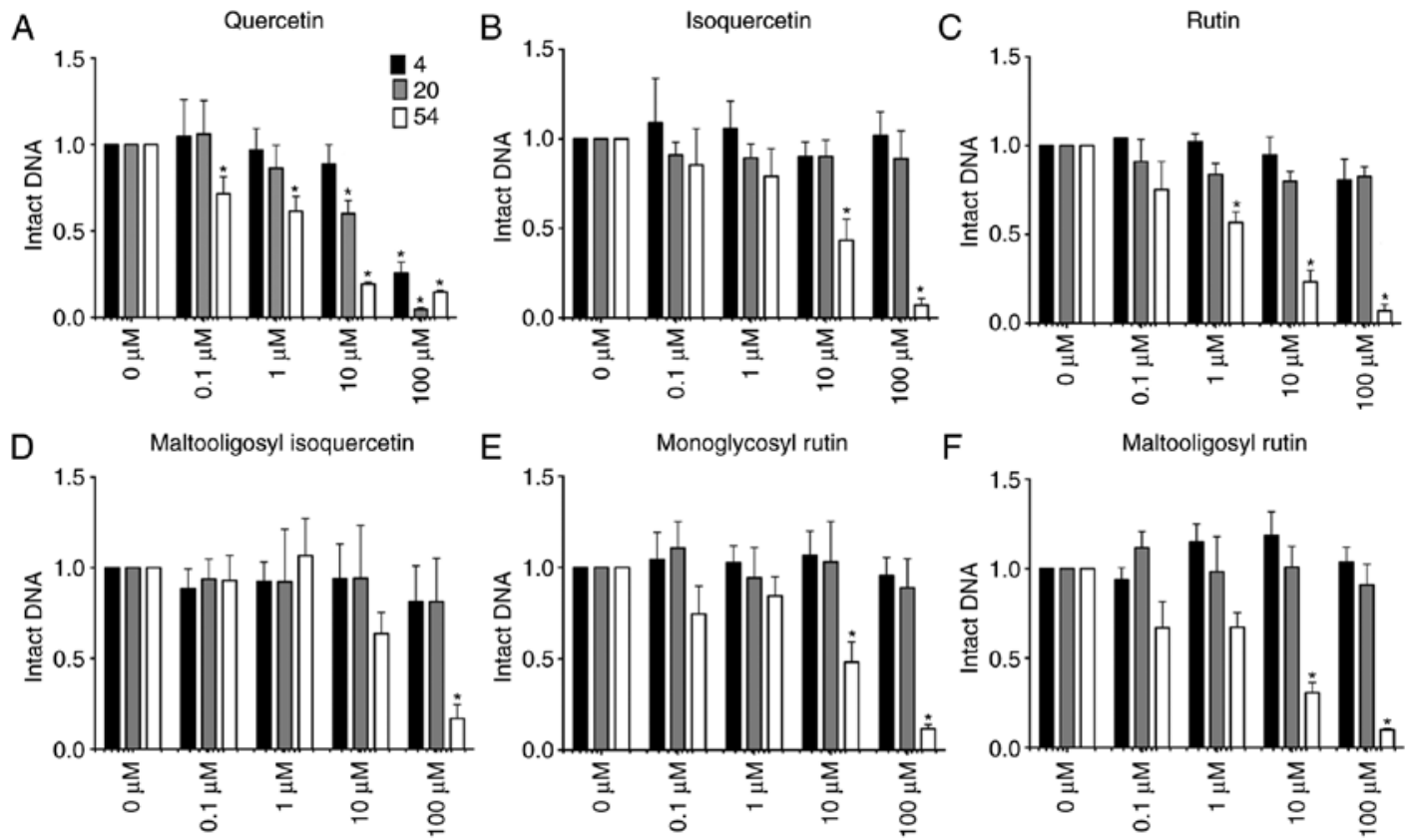

Figure 3. Fraction of intact DNA after 1-h reaction at 4, 20 and $54^{\circ} \mathrm{C}$ for quercetin and its glucosides. (A) Quercetin, (B) isoquercetin, (C) rutin, (D) maltooligosyl isoquercetin, (E) monoglucosyl rutin, and (F) maltooligosyl rutin. Error bars indicate standard error of the means. Three independent experiments were performed.
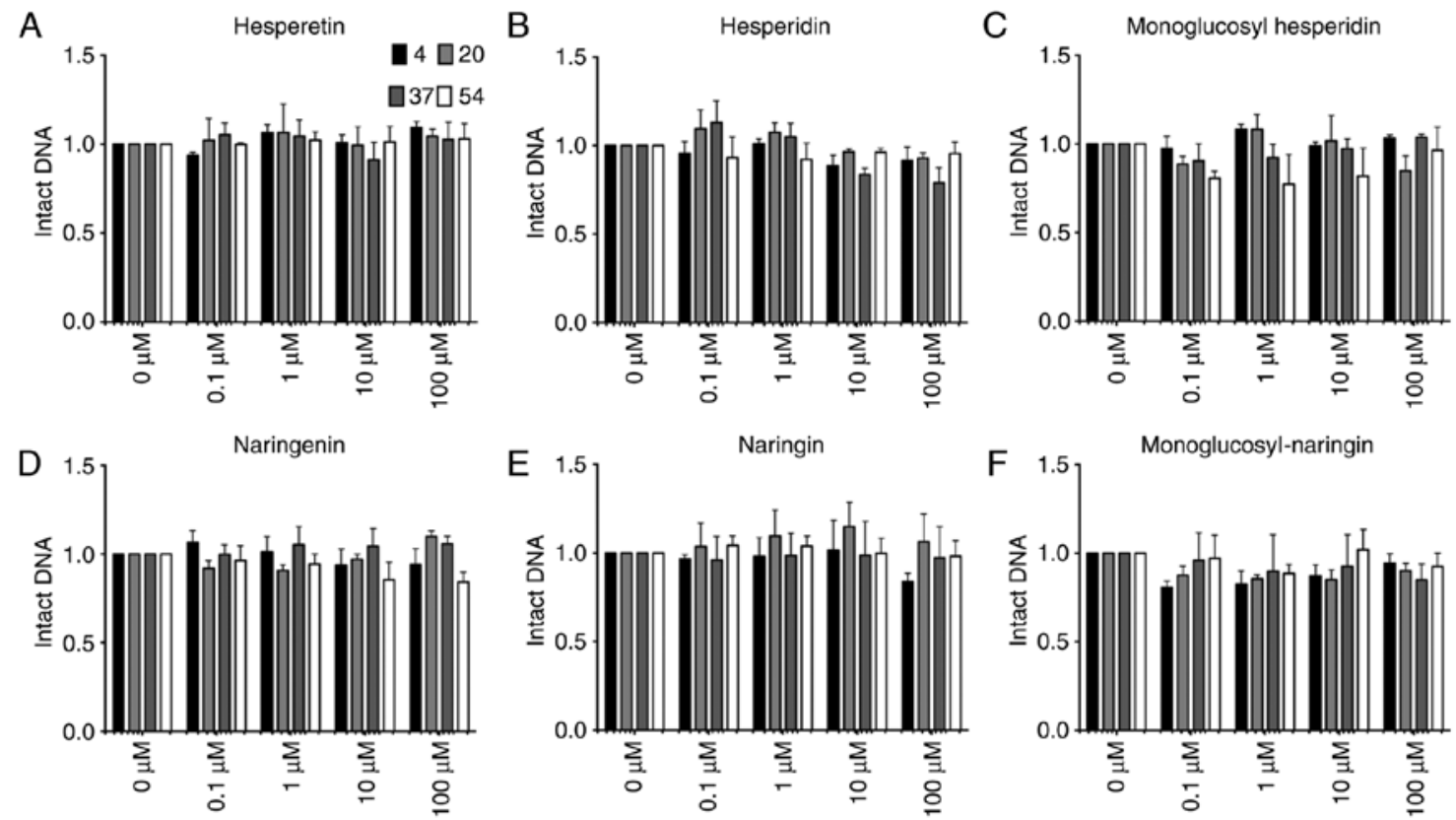

Figure 4. Fraction of intact DNA after 1-h reaction at 4, 20 and $54^{\circ} \mathrm{C}$ for hesperetin, naringenin and their glucosides. Error bars indicate the standard error of the mean. Three independent experiments were performed. (A) Hesperetin, (B) hesperidin, (C) monoglucosyl hesperidin, (D) naringenin, (E) naringin and (F) monoglucosyl naringin.

other flavonoids were reduced by 2-4-fold. Furthermore, quercetin showed statistically significant differences compared with the other flavonoids (ANOVA; $\mathrm{P}<0.0001$ ).

Oxidation of luminol. In addition to the interaction between flavonoids and cupric ions, oxidative capacity analysis was conducted with luminol as a substrate in the presence of flavonoids and cupric ions. Subsequent to mixing, the reduction of the luminescence signal was observed (Fig. 6). Emitted glow signals are associated with not only cupric ion induction, but also generated hydrogen peroxide by flavonoids in the presence of cupric ions. The luminescence signal (arbitrary RLU) was rapidly decreased with an exponentially decreased model for all flavonoids. Quercetin and isoquercetin exhibited the fastest reduction of RLU with a half-life of $0.22 \mathrm{~min}$. In addition, rutin, monoglucosyl-rutin, maltooligosyl-rutin and maltooligosyl-isoquercetin presented intermediate reduction in kinetics with a half-life between $0.28-0.33 \mathrm{~min}$. Naringenin, 

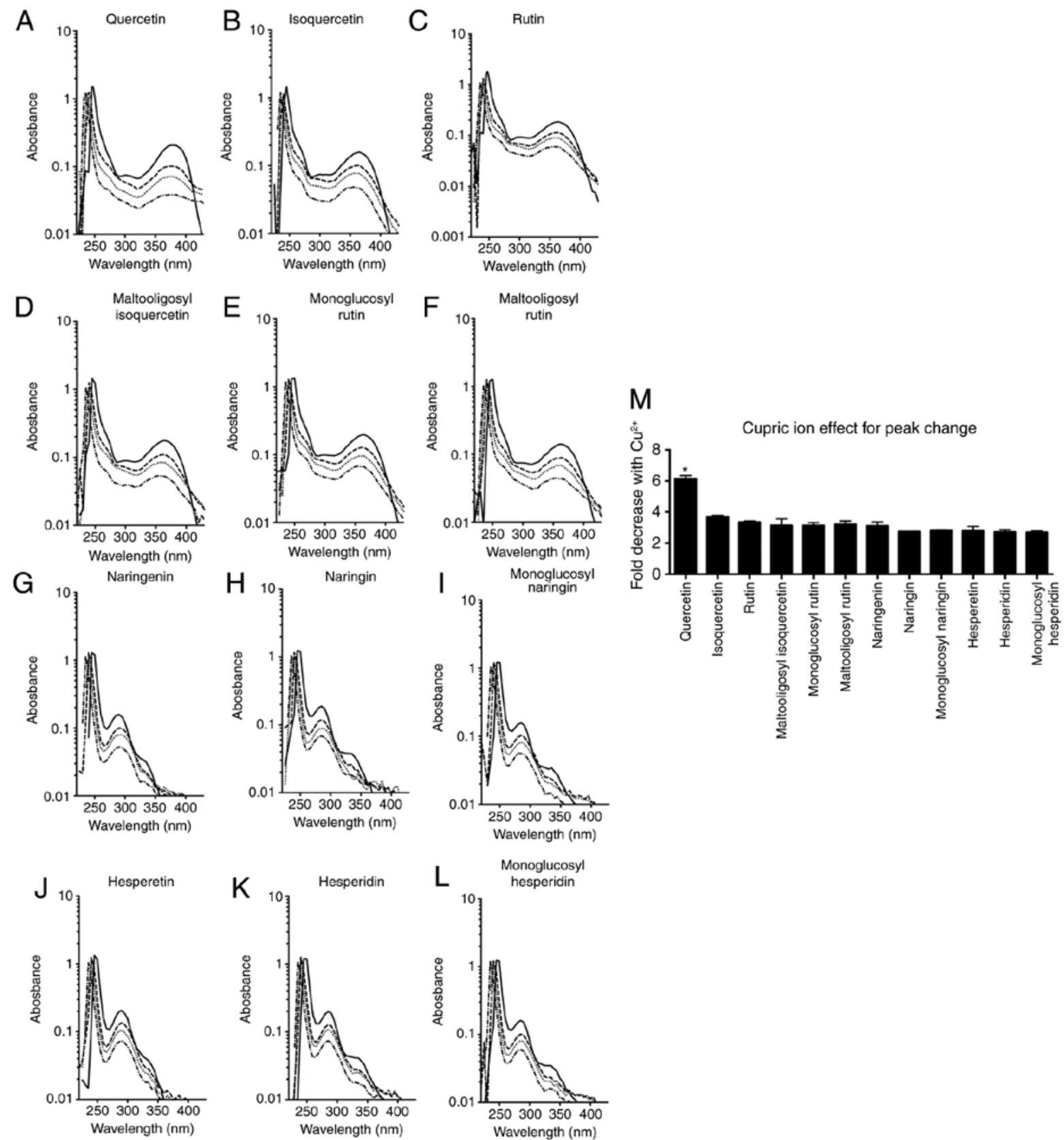

Figure 5. Absorption spectrum changes in the presence of cupric ions. Solid lines indicate the flavonoid only, and the dashed, dotted and dash-dotted lines indicate a flavonoid and cupric ion mixture at a ratio of 2:1, 1:1 and 1:2, respectively. (A) Quercetin, (B) isoquercetin, (C) rutin, (D) maltooligosyl isoquercetin, (E) monoglucosyl rutin, (F) maltooligosyl rutin, (G) naringenin, (H) naringin, (I) monoglucosyl naringin, (J) hesperetin, (K) hesperidin and (L) monoglucosyl hesperidin spectrum changes are shown. (M) Effect of cupric ion on peak changes is displayed. ${ }^{*} \mathrm{P}<0.05$ vs. other flavonoids. Error bars indicate the standard error of the mean. Three independent experiments were performed.

naringin, monoglucosyl-naringin, hesperetin, hesperidin and monoglucosyl-hesperidin also displayed the slowest kinetics with a half-life of 0.35-0.45 min.

\section{Discussion}

Flavonoids with a variety of chemical structures with modified residues exist; for instance, a number of hydroxyl groups are attached to the different positions of benzene rings (21). Depending on the hydroxyl groups present at specific locations, metal ions can bind to flavonoids and induce the Fenton-like reaction, resulting in DNA damage $(11,12)$. DNA double-strand breaks are rare in this event, but single-strand scission and oxidative damage are induced $(11,22)$. This DNA damage may be associated with mutagenesis in the cell culture system following flavonoid reaction with cells in the presence of metals (23).

The present study clearly demonstrated the importance of the specific positions of hydroxyl groups on the flavonoids to induce DNA damage. The results revealed that quercetin was the only flavonoid capable of inducing DNA damage at any of the tested experimental temperatures (Fig. 3). Naringenin and hesperetin, which do not possess a hydroxyl group at the specific positions (the 3-position on the $\mathrm{C}$ ring, and the 3'- and 4'-positions on the $\mathrm{B}$ ring), were incapable of inducing DNA damage (Fig. 4). Furthermore, at low temperatures, 

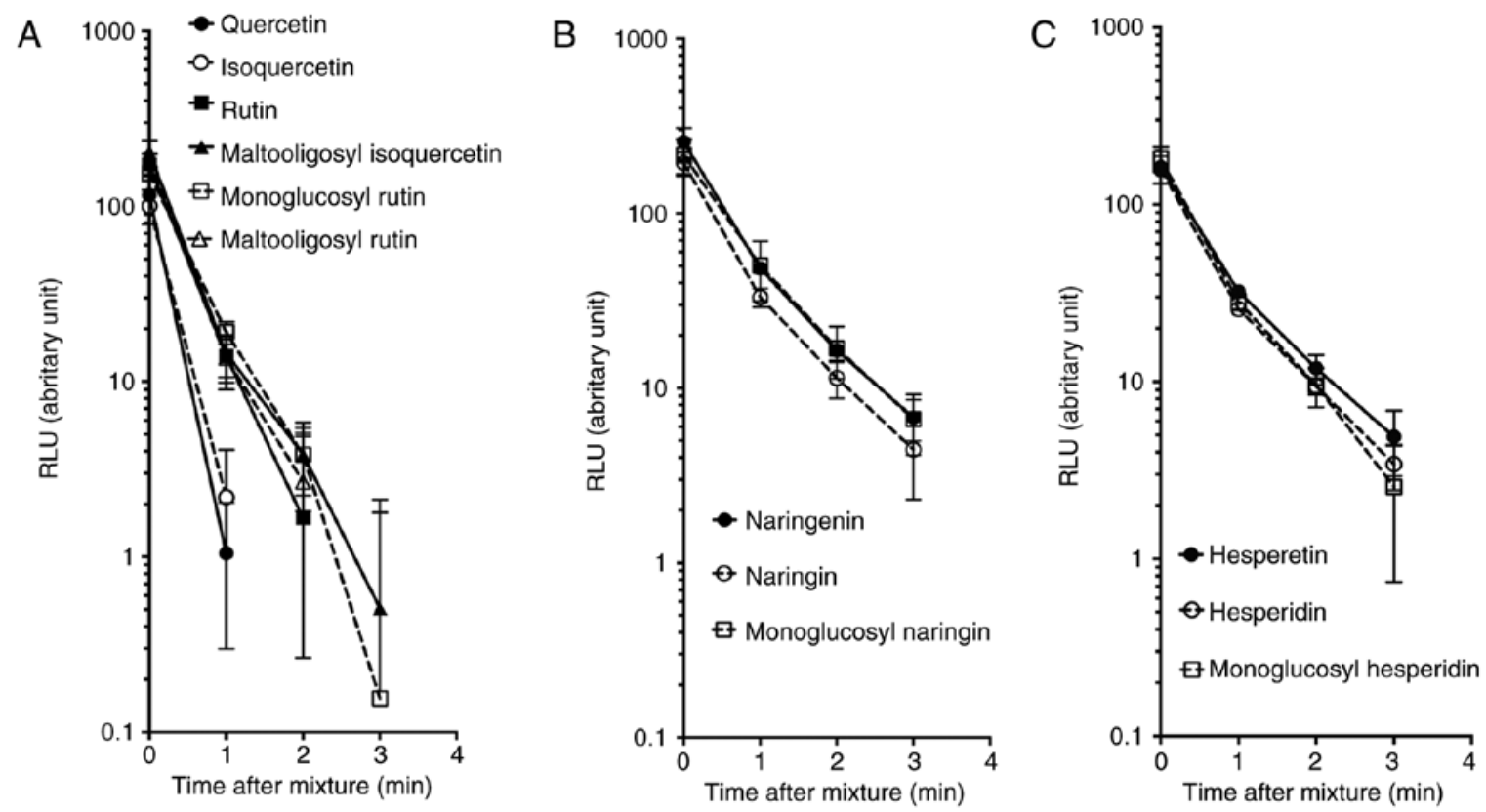

Figure 6. Oxidation of luminol in the presence of hydrogen peroxide, $\mathrm{CuCl}_{2}$ and flavonoids. (A) Quercetin, isoquercetin, rutin, maltooligosyl isoquercetin, monoglucosyl rutin, and maltooligosyl rutin. (B) Naringenin, naringin, and monoglucosyl naringin. (C) Hesperetin, hesperidin, and monoglucosyl hesperidin. Error bars indicate the standard error of the mean values. Three independent experiments were performed. MO, maltooligosyl; MG, monoglucosyl.

the glycosylated flavonoids of quercetin were incapable of inducing DNA breaks (Fig. 3). This suggests that a hydroxyl group at the 3-position on the $\mathrm{C}$ ring of quercetin is the most reactive, inducing DNA damage in a Fenton-like reaction with cupric ions. These findings are in agreement with the previous studies that refer to the possibly significant role of this location (11) in forming a complex with cupric ions to induce DNA damage. It is also worth noting that the hydroxyl group at the 3-position on the $\mathrm{C}$ ring of quercetin has the ability to scavenge radicals (24). The high cupric ion chelating capacity of quercetin (Fig. 5) and fast oxidation of luminol (Fig. 6) were also in agreement with these previous findings.

The structural differences among quercetin, naringenin and hesperetin helped to investigate the impact of hydroxyl groups at the 3'- and 4'-positions on the B ring in the induction of DNA damage. With two hydroxyl groups at the 3'- and 4'-positions on the B ring, flavonoids induced DNA damage at a high temperature, as observed with glycosylated quercetins (including isoquercetin, rutin, maltooligosyl-isoquercetin, monoglucosyl-rutin and maltooligosyl-rutin) (Fig. 3). When one of the two hydroxyl groups was replaced with other residues, high temperature-specific DNA damage was not observed in naringenin and hesperetin. Additionally, hydroxyl groups at the 7-positions on the A ring did not contribute to DNA damage induction based on the observation of the glycosylated naringenin and hesperetin. Previously, it has been reported that the hydroxyl groups of quercetin at the 3'- and 4'-positions on the $\mathrm{B}$ ring have higher radical scavenging effects in comparison with the hydroxyl group at the 3-position on the A ring (24). This may be associated with the temperature-dependent proand anti-oxidant properties of quercetin.

The DNA scission observed in the present study is a result of radical formation from a Fenton-like reaction between specific hydroxyl groups and cupric ions. Temperature-dependent DNA damage between a hydroxyl group at the 3 -position on the $\mathrm{C}$ ring and the hydroxyl groups at the 3'- and 4'-positions on the $\mathrm{B}$ ring may be associated with the amount of radical formation at the different temperatures. In this case, the hydroxyl group at the 3-positions on the $\mathrm{C}$ ring can efficiently produce more radicals compared with the hydroxyl groups at 3 '- and 4'-positions on the B ring in the presence of cupric ions. Another possible mechanism of differential DNA damage is an interaction between the quercetin-cupric ion complex and DNA. The proposed models of the quercetin-cupric ion complex involve a hydroxyl group at the 3-position on the $\mathrm{C}$ ring and hydroxyl groups at the 3'- and 4'-positions on the $\mathrm{B}$ ring. It is possible that the complex formation between quercetin-cupric ion at the 3'- and 4'-positions on the B ring is temperature-dependent.

In conclusion, the present study reported that quercetin induces DNA scissions in the presence of cupric ions in a broad range of temperatures. The hydroxyl group at the 3-position on the $\mathrm{C}$ ring contributes to the temperature-independent DNA scission due to high chelating capacity and oxidative reaction. In addition, the hydroxyl groups at the 3'- and 4'-positions on the $\mathrm{B}$ ring contribute to DNA scission formation in the presence of cupric ions at high temperature.

\section{Acknowledgements}

Not applicable.

\section{Funding}

This study was partially supported by Toyo Sugar refining research contracts (Tokyo, Japan), the Biotechnology Research Center of Libya (Tripoli, Libya) and the Cell and Molecular Biology Program of Colorado State University (Fort Collins, CO, USA). 


\section{Availability of data and materials}

All data generated or analyzed during this study are included in this published article.

\section{Authors' contributions}

TAK conceived and designed the experiment. SE, CS and DJB performed experiments and wrote the manuscript. YA and TAK performed data analysis and critical revision of the article. All authors read and approved the final manuscript.

\section{Ethics approval and consent to participate}

Not applicable.

\section{Consent for publication}

Not applicable.

\section{Competing interests}

The authors declare that they have no competing interests.

\section{References}

1. Havsteen BH: The biochemistry and medical significance of the flavonoids. Pharmacol Ther 96: 67-202, 2002.

2. Yao LH, Jiang YM, Shi J, Tomás-Barberán FA, Datta N, Singanusong R and Chen SS: Flavonoids in food and their health benefits. Plant Foods Hum Nutr 59: 113-122, 2004.

3. Amin A and Buratovich M: The anti-cancer charm of flavonoids: A cup-of-tea will do! Recent Pat Anticancer Drug Discov 2: 109-117, 2007.

4. Yu H, Haskins JS, Su C, Allum A, Haskins AH, Salinas VA, Sunada $S$, Inoue $T$, Aizawa $Y$, Uesaka $M$ and Kato TA: In vitro screening of radioprotective properties in the novel glucosylated flavonoids. Int J Mol Med 38: 1525-1530, 2016.

5. Maeda J,Roybal EJ,Brents CA,UesakaM,Aizawa Y and KatoTA Natural and glucosyl flavonoids inhibit poly(ADP-ribose) polymerase activity and induce synthetic lethality in BRCA mutant cells. Oncol Rep 31: 551-556, 2014.

6. Engen A, Maeda J, Wozniak DE, Brents CA, Bell JJ, Uesaka M, Aizawa $\mathrm{Y}$ and Kato TA: Induction of cytotoxic and genotoxic responses by natural and novel quercetin glycosides. Mutat Res Genet Toxicol Environ Mutagen 784-785: 15-22, 2015.

7. Gaspar J, Rodrigues A, Laires A, Silva F, Costa S, Monteiro MJ, Monteiro C and Rueff J: On the mechanisms of genotoxicity and metabolism of quercetin. Mutagenesis 9: 445-449, 1994.
8. Bjeldanes LF and Chang GW: Mutagenic activity of quercetin and related compounds. Science 197: 577-578, 1977.

9. Brown JP and Dietrich PS: Mutagenicity of plant flavonols in the Salmonella/mammalian microsome test: Activation of flavonol glycosides by mixed glycosidases from rat cecal bacteria and other sources. Mutat Res 66: 223-240, 1979.

10. MacGregor JT and Jurd L: Mutagenicity of plant flavonoids: Structural requirements for mutagenic activity in Salmonella typhimurium. Mutat Res 54: 297-309, 1978.

11. Rahman A, Shahabuddin, Hadi SM, Parish JH and Ainley K: Strand scission in DNA induced by quercetin and $\mathrm{Cu}$ (II): Role of $\mathrm{Cu}(\mathrm{I})$ and oxygen free radicals. Carcinogenesis 10: 1833-1839, 1989.

12. Rahman A, Fazal F, Greensill J, Ainley K, Parish JH and Hadi SM: Strand scission in DNA induced by dietary flavonoids: Role of $\mathrm{Cu}(\mathrm{I})$ and oxygen free radicals and biological consequences of scission. Mol Cell Biochem 111: 3-9, 1992.

13. Liu Y and Guo M: Studies on transition metal-quercetin complexes using electrospray ionization tandem mass spectrometry. Molecules 20: 8583-8594, 2015.

14. Pekal A, Biesaga M and Pyrzynska K: Interaction of quercetin with copper ions: Complexation, oxidation and reactivity towards radicals. Biometals 24: 41-49, 2011.

15. Ríha M, Karlícková J, Filipský T, Jahodár L, Hrdina R and Mladenka P: In vitro copper-chelating properties of flavonoids. Free Radic Biol Med 75 (Suppl 1): S46, 2014

16. Cherrak SA, Mokhtari-Soulimane N, Berroukeche F, Bensenane B, Cherbonnel A, Merzouk $\mathrm{H}$ and Elhabiri M: In vitro antioxidant versus metal ion chelating properties of flavonoids: A structure-activity investigation. PLoS One 11: e0165575, 2016.

17. Bukhari SB, Memon S, Mahroof-Tahir M and Bhanger MI: Synthesis, characterization and antioxidant activity copper-quercetin complex. Spectrochim Acta A Mol Biomol Spectrose 71: 1901-1906, 2009.

18. Yoshino M, Haneda M, Naruse M and Murakami K: Prooxidant activity of flavonoids: Copper-dependent strand breaks and the formation of 8-hydroxy-2'-deoxyguanosine in DNA. Mol Genet Metab 68: 468-472, 1999.

19. Ahsan H and Hadi SM: Strand scission in DNA induced by curcumin in the presence of $\mathrm{Cu}(\mathrm{II})$. Cancer Lett 124: 23-30, 1998

20. Ohashi Y, Yoshinaga K, Yoshioka H and Yoshioka H: Kinetic analysis of the effect of (-)-epigallocatechin gallate on the DNA scission induced by Fe(II). Biosci Biotechnol Biochem 66: 770-776, 2002.

21. Kumar S and Pandey AK: Chemistry and biological activities of flavonoids: An overview. ScientificWorldJournal 2013: 162750, 2013.

22. Yamashita N, Tanemura $\mathrm{H}$ and Kawanishi S: Mechanism of oxidative DNA damage induced by quercetin in the presence of $\mathrm{Cu}(\mathrm{II})$. Mutat Res 425: 107-115, 1999.

23. Carver JH, Carrano AV and MacGregor JT: Genetic effects of the flavonols quercetin, kaempferol, and galangin on Chinese hamster ovary cells in vitro. Mutat Res 113: 45-60, 1983.

24. Chen JW, Zhu ZQ, Hu TX and Zhu DY: Structure-activity relationship of natural flavonoids in hydroxyl radical-scavenging effects. Acta Pharmacol Sin 23: 667-672, 2002. 